\title{
Smart Government - Partizipation und Empowerment der Bürger im Zeitalter von Big Data und personalisierter Algorithmen
}

\author{
Ali Asker Guenduez • Tobias Mettler (iD · Kuno Schedler
}

Eingegangen: 6. Februar 2017 / Angenommen: 15. März 2017

(C) Springer Fachmedien Wiesbaden 2017

Zusammenfassung Anhand zwei konkreter Fallbeispiele beschreibt der vorliegende Artikel anschaulich wie staatliche Behörden und öffentliche Organisationen die heutigen Potenziale der Digitalisierung und integrierter Sensorsysteme zu nutzen versuchen. Unter dem Stichwort „Smart Goverment“ werden neuartige IT-Initiativen lanciert, welche mehr als „E-Government“ auf eine ganzheitliche Vernetzung von physischen, digitalen, öffentlichen und privaten Lebensräumen abzielen. Dabei spielt die aktive und passive Partizipation von Bürgern und anderen Stakeholdern eine wesentliche Rolle. Nur so können die für die algorithmische Entscheidungsfindung notwendigen Daten generiert werden, welche für die personalisierte Interaktion mit Bürgern oder zur real-time Steuerung öffentlicher Infrastrukturen benötigt werden. Der Artikel schließt mit einer kritischen Diskussion über die Möglichkeiten und Grenzen von Smart Government Initiativen und deren Einfluss auf das Privatleben der Bürger und die öffentliche Politikgestaltung.

Schlüsselwörter Algorithmische Entscheidungsfindung - Big Data · Internet of Things · Sensoren · Smart Government

\footnotetext{
A. A. Guenduez $\cdot$ K. Schedler

Institut für Systemisches Management und Public Governance, Universität St. Gallen, Dufourstrasse 40a, 9000 St. Gallen, Schweiz

T. Mettler $(\bowtie)$

Hochschulinstitut für öffentliche Verwaltung (IDHEAP), Universität Lausanne, Rue de la Mouline 28, 1022 Chavannes-près-Renens, Schweiz

E-Mail: tobias.mettler@unil.ch
} 


\title{
Smart Government - Participation and empowerment of citizens in the era of big data and personalized algorithms
}

\begin{abstract}
Based on two practical case studies this article illustrates how governmental authorities and public organizations try to harness the potentials of digitalization and integrated sensor systems. Even more than past e-government intiatives, many projects are launched today under the umbrella term "smart government" aiming at connecting physical, digital, public, and private environments. Active as well as passive participation of citizens and other stakeholders plays a pivotal role for generating the necessary data for algorithmic decision algorithms such that personalized interaction and real-time control of infrastructures are possible. This article closes with a critical discussion about the possibilities and boundaries of smart government initiatives and its possible influence on citizen's private lives and public policy-making.
\end{abstract}

Keywords Algorithmic decision-making · Big data - Internet of Things - Sensors · Smart Government

\section{Innovation im öffentlichen Sektor: Von e-Government zu Smart Government}

Seit Mitte der 90er-Jahre des letzten Jahrhunderts existieren Bestrebungen das schier unerschöpfliche Potential des Internets für die öffentliche Verwaltung nutzbar zu machen. Unter dem Stichwort „Electronic Government“ (E-Government) hielt die zunehmende Digitalisierung Einzug in die öffentliche Verwaltung. Als Ziele dieser Digitalisierung wurden die Erhöhung der Transparenz des Regierungs- und Verwaltungshandelns, die Verbesserung der Rechenschaftspflicht, die Förderung der Partizipation der und Zusammenarbeit mit den Bürgern und anderen Stakeholdern sowie die Steigerung der Effizienz und Effektivität staatlichen Handelns definiert (Schedler et al. 2003; Yildiz 2007). Die bisherige Praxis, sowohl national als auch international, zeigt indessen, dass das Hauptmerkmal des E-Governments darin besteht, den Zugang der Bürger zu öffentlichen Dienstleistungen unter Verwendung neuer Informations- und Kommunikationstechnologien zu vereinfachen. In diesem Sinne wird E-Government im Kern oft als ein „Online gehen“ der Verwaltung verstanden. So definieren die Vereinten Nationen E-Government als ,utilizing the internet and the world-wide-web for delivering government information and services to citizen" (United Nations and American Society for Public Administration 2002). In der Schweiz beispielsweise zeigt sich das Online gehen der Verwaltung in Initiativen und Projekten, welche Namen wie ,e-Steuererklärung“, ,e-Rechnung“, ,e-Ausländerausweis“, ,e-Baugesuch“ oder ,e-Umzug“ tragen. In der einschlägigen Literatur werden für das Phänomen des E-Governments daher Begriffe wie „Virtual State“ oder ,NetState“ verwendet.

Die bisherige Digitalisierung der staatlichen und kommunalen Verwaltung in der Form des E-Governments wird jedoch dem Volumen, der Geschwindigkeit und der Vielfalt des digitalen Fortschrittes nicht mehr gerecht. Außerdem bleibt sie in der 
Regel auf ein Gemeinwesen oder einen Fachbereich beschränkt und vernetzt sich kaum über solche institutionellen Grenzen hinweg.

Neue Ansätze gehen einen Schritt weiter als das E-Government im Sinne des bloßen Online Gehens der öffentlichen Verwaltung. Sie fragen danach, wie das Regieren und Verwalten von Gemeinwesen in Zeiten smarter Technologien und Applikationen, Big Data, Internet of Things und Künstlicher Intelligenz aussehen könnte. Daher sollen neue Government- und Governance Strukturen über bisherige E-Government Initiativen hinausgehen. Neue Prozesse und Formen der Partizipation, die durch den Einsatz neuer Technologien und Applikationen möglich werden, sollen erforscht werden (Gil-Garcia 2012). Diese Erweiterung des E-Government wird als Smart Government bezeichnet. Die bisherigen Definitionen heben vor allem auf die neuen Technologien und Applikationen hervor. So definieren Mellouli, Lune-Reyes und Zhang (2014) das Konzept als ,the extensive use of technology by governments“. Die Anwendung smarter Technologien und Applikationen durch Gemeinwesen heben auch Coe, Paquet und Roy (2001) als Kernelement des Konzepts des Smart Governments hervor. Andere Autoren gehen einen Schritt weiter und rücken Schwerpunkte in den Vordergrund, die mit Smart Government gesetzt werden. Gil-Garcia (2012) fokussiert auf die verbesserte Dienstleistungsqualität und definiert Smart Government als die Nutzung von ,,sophisticated information technologies to interconnect and integrate information, processes, institutions, and physical infrastructure to better serve citizens and communities“. Von Lucke (2016) betont in diesem Zusammenhang ein ,,intelligent vernetztes Regierungs- und Verwaltungshandeln", welches durch die Anwendung neuer Technologien und Applikationen ermöglicht wird, als konstitutives Element des Smart Government. Gil-Garcia, Helbig und Ojo (2014) sehen Smart Government als ein Zusammenspiel neuer Technologien und Innovation im öffentlichen Sektor. Expliziter als in diesen Definitionen heben Harsh und Ichalkaranje (2015) die Bedeutung der verfügbaren Daten hervor, die durch die Verwendung neuer Technologien und Applikationen generiert werden. Nach der Auffassung dieser Autoren verbessert die Analyse großer Daten die Dienstleistungserbringung und ermöglicht es den Regierungen, E-Government in Smart Goverment zu transformieren. Andere Autoren nähern sich dem Konzept aus einer Managementperspektive. So definieren Nam und Pardo (2011) Smart Government als ,a mechanism to create managerial and organizational capabilities for effective use of technological tools and conditions“. Scholl und Scholl (2014) beleuchten schließlich Smart Government als ein umfassendes Konzept intelligenter Verwaltung, indem sie etwa die Transparenz von Regierungsentscheidungen und -handlungen, den offenen Informationsaustausch, die Beteiligung aller Stakeholder, die Verbesserung von Dienstleistungen durch die Verwendung intelligenter Technologien, die Förderung von Innovationen, von Nachhaltigkeit, Wettbewerb und Lebensqualität als konstitutive Elemente hervorheben (Scholl und Scholl 2014). Zusammenfassend lässt sich festhalten, dass Smart Government den Versuch darstellt, neue und innovative Technologien und Applikationen für das Einlösen bereits vom E-Government bekannter Versprechen (z. B. Offenheit, Transparenz, Innovation, Partizipation) einzusetzen. Smarte Regierungen und Verwaltungen wollen die Situation, in der sich die Bürger befinden (z. B. Verkehr, Sicherheit, Gesundheit, Soziales), besser einschätzen und darauf basierend schnell effektive Entscheidungen treffen (Gil-Garcia et al. 
2014). Mit der Betonung von vernetztem Regierungs- und Verwaltungshandeln und einer Managementperspektive rückt eine ganzheitliche und betriebswirtschaftliche Sichtweise in den Vordergrund. Smart Government leitet damit weniger einen Paradigmenwechsel ein, sondern es handelt sich eher um ein sprunghaftes Weiterdenken des E-Governments.

\section{Was braucht es, damit Smart Government,,smart“" wird?}

In der einschlägigen Literatur besteht weitestgehend Konsens darüber, dass Smart Government Initiativen wesentlich zur Lösung heutiger und zukünftiger gesellschaftlicher Herausforderungen beitragen können (Scholl und Scholl 2014; Gil-Garcia et al. 2014). Weniger klar ist jedoch hingegen, inwiefern eine umfangreiche Digitalisierung und Einführung von Sensoren und integrierter Messelektronik eine staatliche Organisation oder Behörde „smarter“ macht.

Tatsache ist, dass Smart Government in der Praxis häufig mit dem Aufbau von leistungsstarken Infrastrukturen gleichgesetzt wird, wie beispielsweise ein intelligentes Stromnetz zur Messung und Regulierung des Energieverbrauchs einzelner Häuser respektive ganzer Ortschaften oder intelligente Parkplatzleitsysteme zum Management der Auslastung unterschiedlicher Parkmöglichkeiten innerhalb einer Region oder Gemeinde. Das „Smarte“ bei solchen Anwendungsfällen ergibt sich durch die Vernetzung der physischen, öffentlichen Infrastruktur (z. B. Abfallcontainer, Ampeln, Parkplatzschranken, Stromzähler). Dies bildet den Grundstein für eine automatisierte Datensammlung, Datenintegration und letztlich einfache Steuerungs-, Regelungs- oder Alarmierungsaufgaben (z. B. ereignisorientierte „wenn A dann B“ Prozeduren). Eine aktive Partizipation durch den Bürger ist für die kollektive Realtime-Steuerung nicht erforderlich.

Während sich durch solche intelligente Infrastrukturen zahlreiche Alltagsprobleme verringern lassen (z. B. Stromsparen, Verkehrsroutenoptimierung), ist damit eine komplexe Entscheidungsfindung, wie zum Beispiel das Vorbereiten politischer Geschäfte oder die Evaluation staatlicher Interventionen, in den seltensten Fällen möglich. Wie in Abb. 1 dargestellt, braucht es hierfür eine Architektur, welche zusätzlich zur öffentlichen auch die private Infrastruktur zur Datensammlung bestmöglich einsetzt und die aktive Partizipation der Bürger vorsieht. Eine Vielzahl unterschiedlicher Quellen (z. B. Smartphones, intelligente Uhren, Mikrocomputer) ermöglicht es, zu einem höheren Detaillierungsgrad der Daten und schließlich zu Informationstransparenz - ein wesentlicher Mehrwert aus Big Data - zu gelangen. Das „Smarte“ bei solchen Anwendungsfällen steckt in der kontext-bezogenen Auswertung einer großen Menge strukturierter und unstrukturierter Daten, welche es selbstlernenden Algorithmen ermöglicht, immer präzisiere Aussagen über bestimmte Sachverhalte und Personen zu machen (contextualized decision-making).

Smart Government wird - anders als das E-Government - bislang nicht im $\mathrm{Zu}$ sammenhang mit formalisierten demokratischen Entscheidungsprozessen gesehen (E-Democracy), hingegen enthält es enormes Potenzial für informelle Partizipation. Die aktive Partizipation der Bürger kann in solchen Anwendungsfällen unterschiedlichste Formen annehmen, u. a. Konzession persönlicher Daten (z. B. physiologische 
Abb. 1 Aktive und passive Partizipation von Bürgern bei Smart Government Initiativen

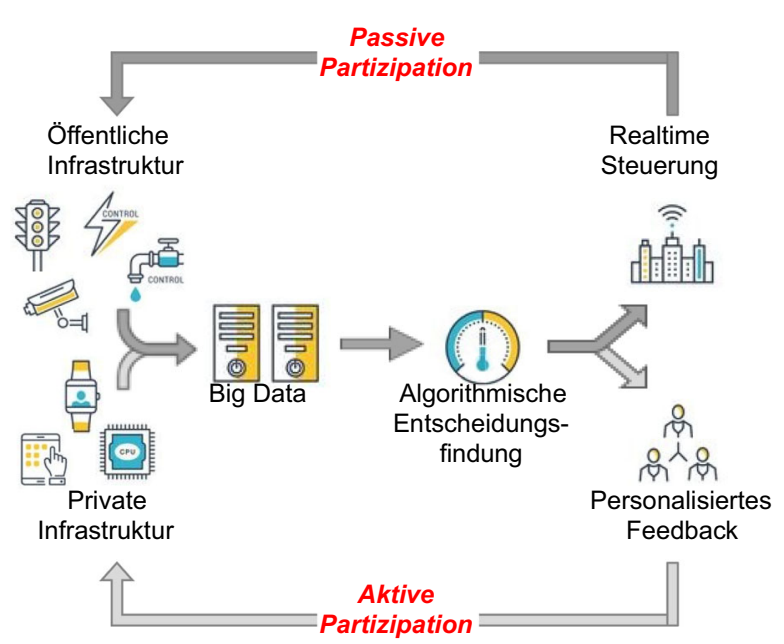

oder genetische Merkmale) zur Verbesserung öffentlicher Forschung und Dienste (citizen science), elektronische Stellungnahme zu aktuellen Anhörungen und behördliche Vorhaben (online petition, e-voting), sowie Teilhabe an der Ideenfindung und Exploration von Innovation in der öffentlichen Verwaltung (online deliberation).

Anders als bei Smart Government Initiativen, die das Ziel verfolgen einfache Steuerungs-, Regelungs- oder Alarmierungsaufgaben zu automatisieren, spielt bei Projekten, welche die Entwicklung kontext-basierter Entscheidungshilfen beabsichtigen, die interaktive und gemeinsame Wertschöpfung eine entscheidende Rolle (cocreation). Wird der Bürger lediglich als „,Datenlieferant“ gesehen (ohne eine Gegenleistung zu erbringen, wie bspw. personalisierte Feedbacks), so kommt die aktive Partizipation schnell zum Erliegen, weil die für die Entwicklung aussagekräftiger Prognosen notwendigen Daten fehlen.

Die aktive Partizipation stellt ein wesentliches Charakteristikum dessen dar, was heute rückblickend als „Wiki government“, „Crowdsourcing“, ,Open government“, „Government 2.0“ oder „We-government“ bezeichnet wird. Die Bedeutung der aktiven Partizipation der Bürger für ein demokratisches Regieren und Verwalten ist bekannt und beruht auf dem Gedanken, dass für Demokratien aktive Bürger besser sind als passive Bürger. Neue Technologien ermöglichen eine neue Form der Interaktion zwischen dem Staat, Bürgern und anderen Stakeholdern. Sie erzeugen Daten, die Auskunft über die Nutzung und Wirksamkeit von Dienstleistungen in wichtigen Politikbereichen wie Verkehr, Gesundheit, Sicherheit oder Landwirtschaft geben. Der große Vorteil dieser Daten liegt darin, dass sie Echtzeitinformationen darstellen und zu trivialen Kosten generiert werden. Sie bieten eine neue Grundlage für die Entscheidungen der Regierung und der Verwaltung und machen eine einfache, bedarfsgerechte und kostengünstige Regelung und Steuerung erst möglich. Im Kontrast zu der aktiven Partizipation lässt sich diese Form der Beteiligung als passive Partizipation bezeichnen (siehe Abb. 1).

Der große Vorteil der durch aktive und passive Partizipation generierten Daten liegt darin, dass sie kombinierbar sind und dadurch auch komplexere Wirkungs- 
zusammenhänge darstellbar werden. Durch Analyse und Auswertung dieser Daten, die unter anderem Hinweise auf das konkrete Verhalten der Bürgerinnen und Bürger liefern, können genauere Erkenntnisse über die Effizienz und Effektivität staatlicher Maßnahmen gewonnen werden (Mergel et al. 2016).

Nachfolgend werden anhand von zwei Fallbeispielen aus der Praxis die Unterschiede zwischen aktiver und passiver Partizipation näher erläutert.

\section{Passive Partizipation am Fallbeispiel „Smart City St.Gallen“}

Die Stadt St.Gallen stellt im deutschsprachigen Raum ein geeignetes Beispiel dar, um den passiven Partizipationszyklus zu veranschaulichen. Von Beginn an zählte die Stadt elektronische Diskussionsforen sowie elektronische Abstimmungen und Wahlen (eDemokratie, ePartizipation) zu den wichtigsten Zielen ihrer E-Government Strategie. Die Digitalisierung wird als eine Möglichkeit gesehen, elektronische Abwicklung und Unterstützung demokratischer Entscheidungsverfahren zu erleichtern. Der aktuelle Stand der Umsetzung des E-Governments zeigt, dass die ursprünglich gesetzten Ziele noch nicht erreicht sind und die Möglichkeiten der Digitalisierung nicht ausgeschöpft werden.

Neuere Digitalisierungsinitiativen, die von den St.Galler Stadtwerken verfolgt werden, gehen einen wesentlichen Schritt weiter als das bisherige E-Government. Ziel der neuen Initiativen ist es, die Möglichkeiten der Digitalisierung einzusetzen. Bei der Nutzung smarter Technologien und Applikationen verfolgt die Stadt einen ganzheitlichen Ansatz. Sie beabsichtigt die Realisierung einer kundenfreundlichen, ökologisch nachhaltigen und energieeffizienten Stadt.

Die Stadt St.Gallen befindet sich auf dem Weg zu einer Smart City. Mit dem Bau und Ausbau des Glasfasernetzes, welches als Nervensystem der Stadt bezeichnet wird, hat sie die Grundlage für eine Smart City geschaffen. Das Glasfasernetz wurde durch eine strahlungsarme LoRa (Long Range) Funktechnologie erweitert. Die LoRa Funktechnologie hat eine große Reichweite und ermöglicht eine ausreichende Durchdringung des städtischen Umfeldes. Glasfasernetz und LoRa Funktechnologie bilden die Infrastruktur, auf die die Stadt aufbaut, um die Möglichkeiten von IoTTechnologien zu nutzen. An diese Infrastruktur knüpfen Sensoren, die an Stromzähler, Straßenlaternen, Lichtsignale, öffentliche Gebäude, Parkplätze oder Fahrzeuge angebracht werden. Die folgende Abb. 2 (St.Galler Stadtwerke 2015) veranschaulicht, wie jeweils kontextbezogene Daten automatisch erhoben, in das Gesamtsystem integriert und für eine intelligente Realtime-Steuerung verarbeitet werden.

In der Stadt St.Gallen stehen heute unterschiedliche IoT-Anwendungen zur Verfügung, die bereits aus anderen Smart City Initiativen bekannt sind (St.Galler Stadtwerke 2017):

- Smart Metering: Intelligente Maßsysteme ersetzen herkömmliche Wasserzähler und übermitteln die Verbrauchsdaten automatisch. Geplant ist, die Anwendung von Smart Metering auf Elektrizität oder Gas auszudehnen.

- Öffentliche Beleuchtung: Intelligente Straßenlaternen erfassen sich nähernde Personen und Fahrzeuge und regulieren die Intensität der Straßenbeleuchtung. 


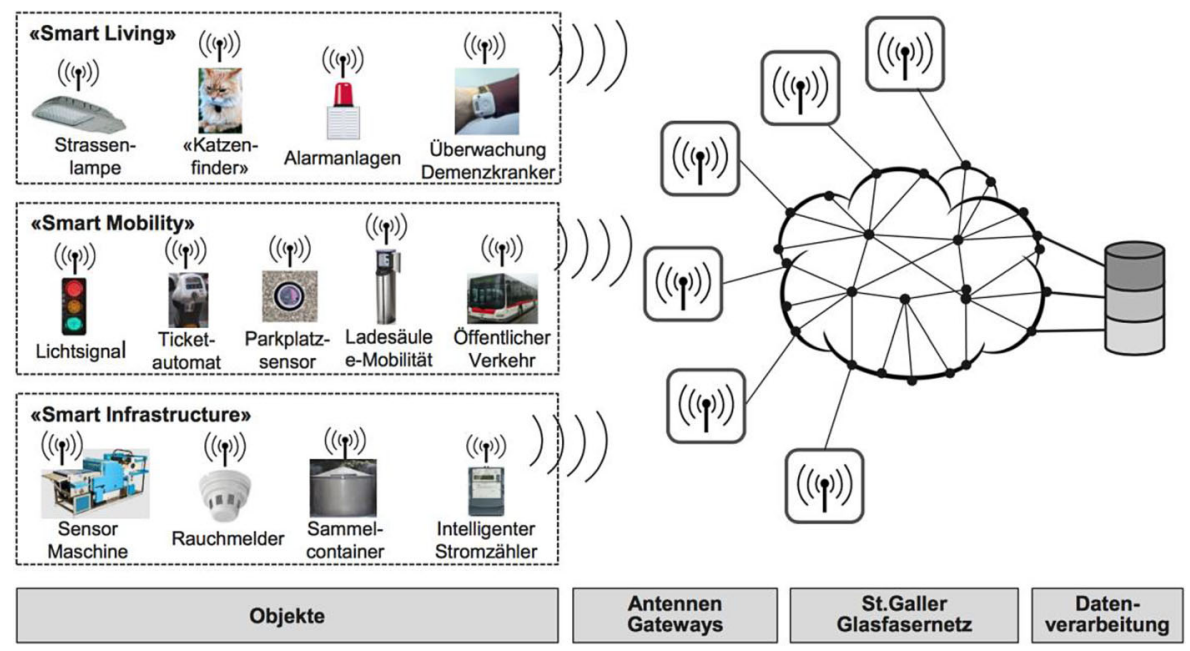

Abb. 2 Technische Grundlagen der passiven Partizipation im Smart City St.Gallen

- Parkplatzbelegung: Freie Parkplätze werden an Autofahrer übermittelt. Dadurch wird der Suchverkehr auf Parkplätzen vermieden.

- Online-Parkkarte: Mit einem Smartphone oder einem ParkingCard-Badge können Fahrer in der Stadt die Parkgebühren bezahlen.

- Füllstandmessung von Unterflurbehältern: Die Füllstände von Abfallbehältern und Sammelcontainern werden automatisch an die Zentrale übermittelt.

- Intelligente Fahrgastinformationen: Öffentliche Transportmittel (Busse und Bahnen) liefern Positions- und Betriebsdaten. Diese sind für die Nutzer an Haltestellen sowie in der App ,Wemlin“ in Echtzeit ersichtlich.

Bei allen aufgeführten Anwendungsfällen werden die sensorgenerierten Daten automatisch erhoben und verarbeitet. In Echtzeit werden Informationen über Nutzung, Effizienz und Wirksamkeit der Dienstleistungen gewonnen. Dadurch wird eine einfache, bedarfsgerechte und kostengünstige Regelung und Steuerung erst möglich. Anders als dies bei Wahlen und Abstimmungen der Fall ist, beruht der Politikzyklus nicht auf einer aktiven politischen Willensäußerung der Bürger, sondern auf deren gesellschaftlicher Teilnahme am städtischen Leben.

\section{Aktive Bürgerpartizipation am Fallbeispiel „DeSearch““}

Ein gutes Beispiel für einen aktiven Partizipationszyklus ist der intelligente Suchdienst „DeSearch“. Das aktuell an der Dualen Hochschule Baden-Württemberg Ravensburg/Friedrichshafen in Kooperation mit der Universität St. Gallen laufende Projekt entwickelt eine datenschutzgerechte Lösung zur Ortung von Personen mit erhöhtem Hilfebedarf (z. B. Menschen mit leichter Demenz). Allein in Deutschland sind heute rund 1,2 Mio. Menschen von einer Form von Demenz betroffen. In Österreich sind es rund 100.000, respektive 110.000 in der Schweiz. Dementsprechend 
groß ist das Interesse von staatlichen und anderen gemeinnützigen Institutionen sich dieser gesellschaftspolitisch bedeutsamen Problematik anzunehmen. Dennoch bleiben demenzkranke Menschen vielfach mit der Bewältigung ihres Alltags im Stich gelassen. Zudem kann sich ein Großteil der Betroffenen insbesondere eine stationäre Pflege finanziell kaum leisten, was zur Folge hat, dass heute häufig Angehörige (ohne pflegerische Kenntnisse und Hilfsmittel) einen großen Teil der Betreuung übernehmen.

Ein großes Problem ist die Weglauftendenz demenzerkrankter Menschen. Deshalb werden im klinischen Umfeld solche Personen häufig mit GPS-Tracker ausgestattet, was eine permanente, jedoch nicht zwingend datenschutzgerechte Überwachung ermöglicht. DeSearch unterscheidet sich von existierenden Lösungen insofern dadurch, dass nicht unablässig überwacht, sondern lediglich im Bedarfsfall ein knopfgroßer Bluetooth-Sender (vgl. Abb. 3a), der beispielsweise in Kleider oder Schuhe eingenäht werden kann, eingeschaltet wird (z. B. durch Meldung eines Angehörigen oder Pflegenden). Der Verzicht der permanenten GPS-Überwachung kommt allerdings mit dem Preis der relativ geringen Reichweite des Bluetooth-Senders. Dem wird durch zwei Maßnahmen entgegengewirkt: Da das Langzeitgedächtnis bei demenzerkrankten Menschen meist noch aktiv ist, verwenden diese häufig Busstationen und andere neuralgische Stellen als Orientierungshilfen und Ruhepunkte. Dementsprechend wäre es analog dem vorherigen Fallbeispiel möglich, fixe Örtlichkeiten (z. B. Bahnhöfe, Marktplätze, Bushaltestellen) wie auch mobile Elemente (z. B. Busse, Taxis) mit DeSearch Empfangsstationen auszustatten (vgl. Abb. 3b). Ein jedoch weit größerer Zugewinn an Reichweite wird durch die aktive Partizipation von involvierten Bürgern erwartet. Durch die Installation einer einfachen App werden Smartphones zu mobilen Empfangsstationen, welche den Standort eine in der Nähe befindliche hilfebedürftige Person dem Auslöser der Suchaktion, der Nothilfezentrale, wie auch dem involvierten Bürger selbst weitergibt (vgl. Abb. 4). Dadurch wäre eine aktive Bürgerhilfe möglich und könnte die Suchkosten von hilflos umherirrenden Personen minimieren. Das aktuell in der Evaluation befindliche DeSearch wird zeigen, ob Bürger tatsächlich gewillt sind ihre private Infrastrutkur für gemeinnützige Aktionen, wie beispielsweise für die Personensuche, zur Verfügung zu stellen.

\section{Implikationen von Smart Government}

Smart Government ist ein Phänomen, das sich noch im Entstehen befindet. Was unter diesem Konzept theoretisch und empirisch zu verstehen ist, ist nach wie vor Gegenstand von Diskussionen. Fest steht, dass Smart Government auf aktiver und passiver Partizipation von Bürgern und anderen Stakeholdern beruht. Nur so können die nötigen Informationen generiert, das Potential innovativer Technologien und Applikationen erschöpft und die beabsichtigten Wirkungen erreicht werden.

Die Partizipation gibt den Bürgern und anderen Anspruchsgruppen die Möglichkeit, Einfluss auf die Politikgestaltung zu nehmen. Das Fallbeispiel „Stadt St.Gallen“ illustriert das Potential von smarten IoT-Technologien. Durch das Fahren auf der Straße, das Parkieren in öffentlichen Parkplätzen oder durch die Versorgung von 

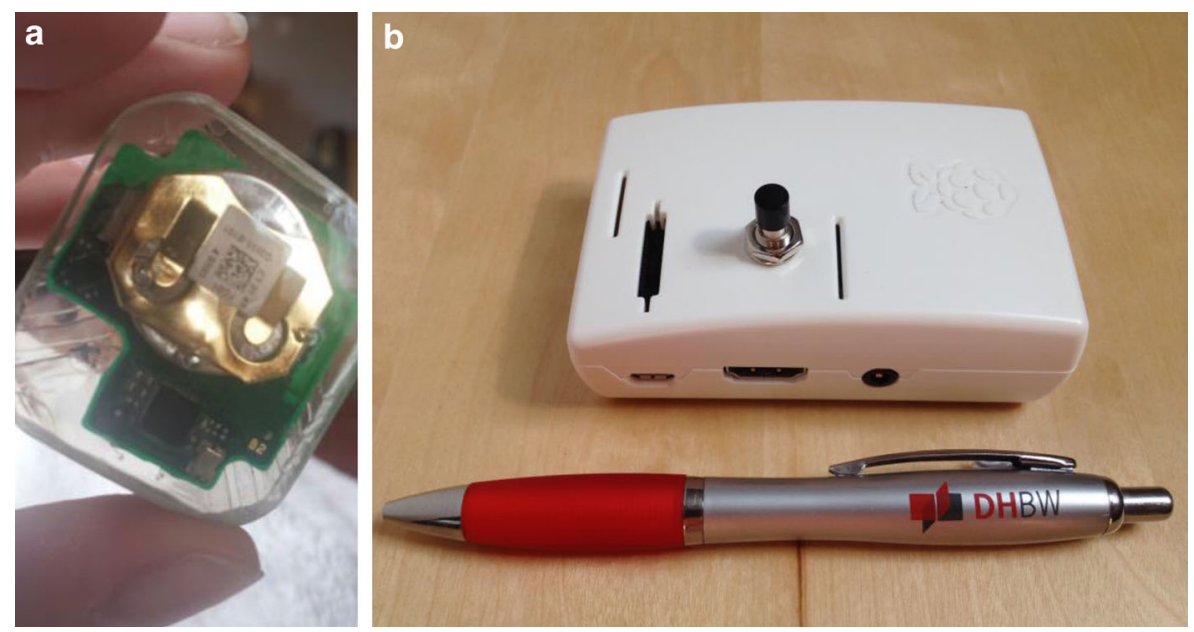

Abb. 3 Komponenten von DeSearch. a DeSearch Bluetooth-Sender, b DeSearch Empfangsstation

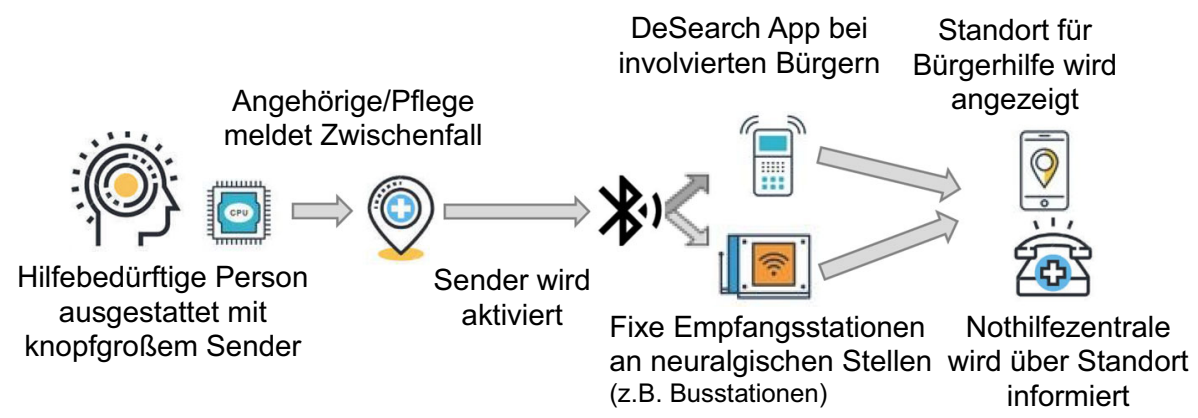

Abb. 4 Aktive Partizipation der Bürger bei DeSearch

Abfällen, kurz durch die Teilhabe am städtischen Leben, partizipieren breite Bevölkerungsschichten implizit und beeinflussen die Politikgestaltung. Die passive Partizipation ergänzt die klassischen Formen der Partizipation (z. B. Wahlen oder Abstimmungen) in vielerlei Hinsicht: Die passive Partizipation ist für den Bürger zeitschonender und für den Staat kostengünstiger. Zudem birgt sie ein demokratisches Potential. Sie integriert auch die von den klassischen Beteiligungsformen ausgeschlossenen Bevölkerungsschichten wie etwa Ausländer und Minderjährige sowie die Nichtwähler am politischen Willensbildungsprozess. Allerdings birgt sie auch ein erhebliches Potenzial, die eigenen Daten aus der Hand zu geben. Ein adäquater Schutz der Daten ist daher zwingend.

Das Fallbeispiel „DeSearch“ zeigt eingehend, wie private Infrastrukturen (Smartphones involvierter Bürger) für öffentliche Aufgaben, wie beispielsweise die Suche vermisster Personen genutzt werden kann. Dadurch eröffnen sich zwar neue Möglichkeiten, jedoch zugleich auch mehrere kritische Fragestellungen. Die vielleicht größte Chance besteht in der Privatisierung öffentlicher Aufgaben. Während im Falle der bisherigen Privatisierungen die Motivation zur Mitwirkung auf der Seite der 
Privaten klar ist, ist die Motivation im Falle der Bürger nicht so einfach gegeben. Mit Schaffung von positiven Stimuli (z. B. monetäre Anreize, Hervorhebung des öffentlichen Interesses) können die Bürger dazu bewegt werden, ihre Infrastruktur zur Verfügung zu stellen.

Die aktive und die passive Partizipation sorgen dafür, dass die IoT-Projekte auch eine entsprechende Wirkung im öffentlichen Sektor erzielen. Die generierten Daten sind das Fundament und zugleich die Achillesferse vom Smart Government. Verschwindende Grenzen zwischen dem, was „privat “ und dem was „öffentlich“ ist, lassen Diskussionen über einen neuen Gesellschaftsvertrag entflammen. Befürchtungen, dass die Privatsphäre durch unbefugten Zugriff auf Daten verletzt wird oder, dass Daten von Regierungen für mehr als zur Politikgestaltung verwendet werden und zu einer staatlichen Überwachung führen, sind groß. Noch wurden nicht alle kritischen Fragen zufriedenstellen beantwortet. Welche Daten dürfen vom wem, in welcher Frequenz erhoben, wie lange archiviert und zu welchen Zwecken analysiert und ausgewertet werden? Die Befugnisse müssen klar zugewiesen, die Grenzen der Privatsphäre geachtet, die sichere Archivierung gewährleistet und eine anonymisierte Analyse und Auswertung der Daten sichergestellt werden. Für eine smarte Regierung und Verwaltung besteht die große Herausforderung darin, das Vertrauen der Bürger und anderer Stakeholder dauerhaft zu gewinnen. Zu diesem Zweck müssen die Bedenken innerhalb der Bevölkerung identifiziert und die Bürger bestmöglich informiert werden. Offene Diskussionen über gewisse „Ausgleichsmechanismen“ für die bestehenden und aufkommenden Spannungsfelder (Sicherheit vs. Datenschutz; Kontrolle vs. Freiheit; Funktionalität vs. Individualität usw.) und der Abbau von Informationsasymmetrien zwischen Staat, Bürgern und anderen Stakeholdern sind von grundlegender Bedeutung. Informierte und involvierte Bürger sind elementar zur Auslotung und Fixierung dieser Grenzen.

Danksagung An dieser Stelle sollen Prof. Dr. Michael Bächle, Prof. Dr. Stephan Daurer und Prof. Dr. Andreas Judt von der DHBW Ravensburg/Friedrichshafen sowie Dr. Ivo Schillig erwähnt, und Ihnen für die aktive Zusammenarbeit und Unterstützung gedankt werden.

\section{Literatur}

Coe A, Paquet G, Roy J (2001) E-governance and smart communities - a social learning challenge. Soc Sci Comput Rev 19(1):80-93

Gil-Garcia JR (2012) Towards a smart State? Inter-agency collaboration, information integration, and beyond. Inf Polity 17(1):269-280

Gil-Garcia JR, Helbig N, Ojo A (2014) Being smart: emerging technologies and innovation in the public sector. Gov Inf Q 31:I1-I8

Harsh A, Ichalkaranje N (2015) Transforming e-government to smart government: a South Australian perspective. In: Jain L, Patnaik S, Ichalkaranje N (Hrsg) Intelligent computing, communication and devices. advances in intelligent systems and computing. Springer, New Delhi, S 9-16

Mellouli S, Luna-Reyes LF, Zhang J (2014) Smart government, citizen participation and open data. Inf Polity 19(1):1-4

Mergel I, Rethemeyer RK, Kimberley I (2016) Big data in public affairs. Public Adm Rev 76(6):928-937

Nam T, Pardo TA (2011) Smart city as urban innovation: focusing on management, policy, and context. Proceedings of the 5th International Conference on Theory and Practice of Electronic Governance, Tallinn, S 185-194

Schedler K, Summermatter L, Schmidt B (2003) Electronic Government einführen und entwickeln. Von der Idee zur Praxis. Paul Haupt, Bern, Stuttgart, Wien 
Scholl HJ, Scholl MC (2014) Smart governance: a roadmap for research and practice. Proceedings of the 2014 iConference, Berlin, S 163-176

United Nations und American Association for Public Administration (2002) Benchmarking e-government: a global perspective. UN Publications, New York

St.Galler Stadtwerke (2015) St.Galler Smartnet: „Eine smarte Stadt braucht ein intelligentes Netz“. Retrieved from https://www.stadt.sg.ch/news/14/2015/06/smartnet/_jcr_content/Par/downloadlist/ DownloadListPar/download_1.ocFile/20150630_MK\%20vom\%201\%20Juli\%202015_Peter\%20St $\% \mathrm{C} 3 \%$ A4ger\%20Smartnet.pdf

St.Galler Stadtwerke (2017) Smart City St.Galler Smartnet- intelligent vernetzt. Retrieved from http:// www.sgsw.ch/home/telecom/geschaeft-telecom.html

Von Lucke J (2016) Deutschland auf dem Weg zum Smart Government - Was Staat und Verwaltung von der vierten industriellen Revolution, von Disruptionen, vom Internet der Dinge und dem Internet der Dienste zu erwarten haben. Verwalt Manag 22(4):171-186

Yildiz M (2007) E-government research: Reviewing the literature, limitations, and ways forward. Gov Inf Q 24(3):646-665 